\title{
Delta Variants of SARS-CoV-2 Cause Significantly Increased Vaccine Breakthrough COVID-19 Cases in Houston, Texas
} Check for updates

\author{
Paul A. Christensen,${ }^{* \dagger}$ Randall J. Olsen,${ }^{* \dagger}$ S. Wesley Long, ${ }^{* \dagger}$ Sishir Subedi, ${ }^{*}$ James J. Davis, ${ }^{\ddagger \S}$ Parsa Hodjat, ${ }^{*}$
} Debbie R. Walley, ${ }^{*}$ Jacob C. Kinskey, ${ }^{*}$ Matthew Ojeda Saavedra, ${ }^{*}$ Layne Pruitt, ${ }^{*}$ Kristina Reppond, ${ }^{*}$ Madison N. Shyer, ${ }^{*}$ Jessica Cambric, ${ }^{*}$ Ryan Gadd, ${ }^{*}$ Rashi M. Thakur, ${ }^{*}$ Akanksha Batajoo, ${ }^{*}$ Regan Mangham, ${ }^{*}$ Sindy Pena, ${ }^{*}$ Trina Trinh, ${ }^{*}$ Prasanti Yerramilli, ${ }^{*}$ Marcus Nguyen, ${ }^{\ddagger \S}$ Robert Olson, ${ }^{\ddagger}$ Richard Snehal, ${ }^{*}$ Jimmy Gollihar, ${ }^{*}$ and James M. Musser ${ }^{* \dagger}$

\begin{abstract}
From the Laboratory of Human Molecular and Translational Human Infectious Diseases, * Center for Infectious Diseases, Houston Methodist Research Institute and Department of Pathology and Genomic Medicine, Houston Methodist Hospital, Houston, Texas; Department of Pathology and Laboratory Medicine, ${ }^{\dagger}$ Weill Cornell Medical College, New York, New York; Consortium for Advanced Science and Engineering, ${ }^{\ddagger}$ The University of Chicago, Chicago, Illinois; Computing, Environment and Life Sciences, ${ }^{\S}$ Argonne National Laboratory, Lemont, Illinois; and DEVCOM Army Research Laboratory-South, ${ }^{\circledR}$ Austin, Texas
\end{abstract}

\author{
Accepted for publication \\ October 26, 2021. \\ Address correspondence to \\ James M. Musser, M.D., Ph.D., \\ Department of Pathology and \\ Genomic Medicine, Houston \\ Methodist Research Institute, \\ 6565 Fannin St., Suite B490, \\ Houston, TX 77030. E-mail: \\ jmmusser@houstonmethodist. \\ org.
}

\begin{abstract}
Genetic variants of severe acute respiratory syndrome coronavirus 2 (SARS-CoV-2) have repeatedly altered the course of the coronavirus disease 2019 (COVID-19) pandemic. Delta variants are now the focus of intense international attention because they are causing widespread COVID-19 globally and are associated with vaccine breakthrough cases. We sequenced 16,965 SARS-CoV-2 genomes from samples acquired March 15, 2021, through September 20, 2021, in the Houston Methodist hospital system. This sample represents $91 \%$ of all Methodist system COVID-19 patients during the study period. Delta variants increased rapidly from late April onward to cause $99.9 \%$ of all COVID-19 cases and spread throughout the Houston metroplex. Compared with all other variants combined, Delta caused a significantly higher rate of vaccine breakthrough cases $(23.7 \%$ for Delta compared with $6.6 \%$ for all other variants combined). Importantly, significantly fewer fully vaccinated individuals required hospitalization. Vaccine breakthrough cases caused by Delta had a low median PCR cycle threshold value (a proxy for high virus load). This value was similar to the median cycle threshold value for unvaccinated patients with COVID-19 caused by Delta variants, suggesting that fully vaccinated individuals can transmit SARS-CoV-2 to others. Patients infected with Alpha and Delta variants had several significant differences. The integrated analysis indicates that vaccines used in the United States are highly effective in decreasing severe COVID-19, hospitalizations, and deaths. (Am J Pathol 2022, 192: 320-331; https://doi.org/10.1016/j.ajpath.2021.10.019)
\end{abstract}

Delta variants of concern (VOCs) of severe acute respiratory syndrome coronavirus 2 (SARS-CoV-2) such as B.1.617.2, AY.3, and AY.4 are the focus of intense international concern because they are causing widespread coronavirus disease 2019 (COVID-19) in the United States, Southeast Asia, Europe, and elsewhere (CDC, https://www.cdc.gov/ coronavirus/2019-ncov/cases-updates/variant-surveillancel variant-info.html, last accessed August 18, 2021; GOV.UK, https://www.gov.uk/government/collections/new-sars-cov-2variant, last accessed August 18, 2021). ${ }^{1}$ For example,
Delta has replaced the Alpha variant in the United Kingdom, previously the cause of virtually all COVID-19

\footnotetext{
Supported by the Houston Methodist Academic Institute Infectious Diseases Fund and supported in whole or in part with federal funds from the National Institute of Allergy and Infectious Diseases, NIH, Department of Health and Human Services (75N93019C00076, J.J.D. and R.O.).

P.A.C., R.J.O., and S.W.L. contributed equally to this work.

Disclosures: None declared.

The findings and conclusions in this article are those of the authors and do not necessarily reflect the views of the US Army.
} 
cases in that country (World Health Organization, https:// www.who.int/publications/m/item/weekly-epidemiologicalupdate-on-covid-19-13-july-2021, last accessed August 18, 2021; Office for National Statistics, https://www.ons. gov.uk/peoplepopulationandcommunity/healthandsocial care/conditionsanddiseases/bulletins/coronaviruscovid 19infectionsurveypilot/9july2021, last accessed August 18, 2021). Vaccine breakthrough cases caused by SARSCoV-2 variants have also become of considerable public health and biomedical concern worldwide. ${ }^{2-6}$

To study Delta spread and vaccine breakthrough cases in metropolitan Houston, 16,965 SARS-CoV-2 genomes were sequenced from patient samples acquired March 15, 2021, through September 20, 2021, using an NovaSeq 6000 instrument (Illumina, San Diego, CA) and with methods as previously described. ${ }^{7,8}$ This period includes the time from initial identification of Delta-related VOCs in late April in our large Houston Methodist health care system until Delta VOCs continuously caused the supermajority $(99.9 \%)$ of all new cases. The sequenced sample is $91 \%$ of the 18,736 total COVID-19 cases diagnosed in our health system during the study period. The characteristics of patients infected with Alpha and Delta VOCs were also compared, an analysis that identified several significant differences between patients infected with these two variants.

\section{Materials and Methods}

\section{Patient Specimens}

Specimens were obtained from registered patients at Houston Methodist hospitals, associated facilities (eg, urgent care centers), and institutions in the Houston metropolitan region that use our laboratory services. The great majority of individuals had signs or symptoms consistent with COVID-19. For analyses focusing on the Delta family variants, a comprehensive sample of genomes obtained from March 15, 2021, through September 20, 2021, was used. This time frame was chosen because it represents the period during which the downturn of our third wave was occurring and soon after the first Delta variant case was identified in our health care system. Subsequently, Delta variants increased rapidly to continuously cause $99.9 \%$ of all new cases. The study included 16,965 unique patients identified in this time frame for whom we had SARS-CoV-2 genome sequences. For analyses comparing features of patients infected with the Delta variants and Alpha VOC, a comprehensive sample of genomes obtained from January 1 , 2021, through September 20, 2021, was used. This time frame represents the period during which the first Alpha variant case was identified in our health care system. This VOC increased rapidly and peaked, and then decreased to cause $<1 \%$ of all cases in the region. This part of the study included 28,560 unique patients identified in the period from January 1, 2021, through September 20, 2021. The work was approved by the Houston Methodist Research Institute Institutional Review Board (IRB1010-0199).

\section{SARS-CoV-2 Molecular Diagnostic Testing}

Specimens obtained from symptomatic patients with a suspicion for COVID-19 were tested in the Molecular Diagnostics Laboratory at Houston Methodist Hospital using assays granted Emergency Use Authorization from the US Food and Drug Administration (US Food and Drug Administration, https://www.fda.gov/medical-devices/emergencysituations-medical-devices/faqs-diagnostic-testing-sarscov-2\#offeringtests, last accessed June 7, 2021). Multiple molecular testing platforms were used, including the COVID-19 test or RP2.1 test with BioFire Film Array instruments (BioFire Diagnostics, Salt Lake City, UT), the Xpert Xpress SARS-CoV-2 test using Cepheid GeneXpert Infinity or Cepheid GeneXpert Xpress IV instruments (Cepheid, Sunnyvale, CA), the Cobas SARS-CoV-2 \& Influenza A/B Assay using the Roche Liat system (Roche Diagnostics, Indianapolis, IN), the SARS-CoV-2 Assay using the Hologic Panther instrument (Hologic, Marlborough, MA), the Aptima SARS-CoV-2 Assay using the Hologic Panther Fusion system (Hologic), the Cobas SARS-CoV-2 test using the Roche 6800 system, and the SARS-CoV-2 assay using Abbott Alinity $\mathrm{m}$ instruments (Abbott Molecular, Des Plaines, IL). Virtually all tests were performed on material obtained from nasopharyngeal swabs immersed in universal transport media; oropharyngeal or nasal swabs, bronchoalveolar lavage fluid, or sputum treated with dithiothreitol were sometimes used. Standardized specimen collection methods were used (Vimeo, https://vimeo.com/396996468/ 2228335d56, last accessed June 7, 2021).

\section{SARS-CoV-2 Genome Sequencing}

Libraries for whole SARS-CoV-2 genome sequencing were prepared according to version 3 or version 4 (ARTIC Network, https://community.artic.network/t/sars-cov-2version-4-scheme-release/312, last accessed August 19, 2021) of the ARTIC nCoV-2019 sequencing protocol. The semi-automated workflow, described previously, ${ }^{7,8}$ used BioMek i7 liquid handling workstations (Beckman Coulter Life Sciences, Indianapolis, IN) and MANTIS automated liquid handlers (Formulatrix, Bedford, MA). Short sequence reads were generated with a NovaSeq 6000 instrument (Illumina). For continuity of the epidemiologic analysis in the study period, some genome sequences reported in a recent publication were also included. ${ }^{8}$

\section{SARS-CoV-2 Genome Sequence Analysis and Identification of Variants}

Viral genomes were assembled with the BV-BRC SARS-Cov2 assembly service (BV-BRC, https://www. 
bv-brc.org/app/ComprehensiveSARS2Analysis,

last accessed June 7, 2021; requires registration). The One Codex SARS-CoV-2 variant calling and consensus assembly pipeline was used to assemble all sequences (GitHub, https://github.com/onecodex/sars-cov-2.git, last accessed June 7,2021 ) using default parameters and a minimum read depth of 3. Briefly, the pipeline uses seqtk version 1 . 3-r116 for sequence trimming (GitHub, https://github.com/ lh3/seqtk. git, last accessed June 7, 2021); minimap version 2.1 for aligning reads against reference genome WuhanHu-1 (NC_045512.2); samtools version 1.11 for sequence and file manipulation; and iVar version 1.2.2 for primer trimming and variant calling. Genetic lineages, VOCs, and variants of interest (VOIs) were identified based on genome sequence data and designated by Pangolin version 3.1.11 with pangoLEARN module 2021-08-09 (Pangolin, https:// cov-lineages.org/resources/pangolin.html, last accessed August 18, 2021). Genome data used in this study have been deposited to GISAID (www.gisaid.org). Accession numbers for the genomes are presented in Supplemental Table S1.

There is a known problem with the ARTIC V3 primer set that results in lack of detection or ambiguity of the G142D and D950N single nucleotide variants, even though these amino acid changes (142D and 950N) are present in the overwhelming majority of B.1.617.2 Delta variant isolates (ARTIC Network, https://community.artic. network/t/sars-cov-2-version-4-scheme-release/312, last accessed September 1, 2021; Pangolin, https:/loutbreak. info/compare-lineages? pango $=$ B.1.617.2\&gene $=S \&$ threshold $=0.2$, last accessed September 1, 2021). After transitioning to ARTIC V4, all Delta variant strain haplotypes were seen to contain both 142D and $950 \mathrm{~N}$ polymorphisms except for four isolates, two of which lacked 142D and two of which lacked $950 \mathrm{~N}$. A recent analysis confirmed the previously identified ARTIC V3 problem. ${ }^{9}$ Thus, for the Delta isolates presented here, virtually all samples sequenced with ARTIC V3 are presumed to have G142D and D950N.

\section{Patient Metadata and Geospatial Analysis}

Patient metadata (Tables 1, 2, and 3) were acquired from the electronic medical record by using standard informatics methods. Patient home address zip codes were used to visualize the geospatial distribution of spread for each VOC and VOI. Figures were generated with Tableau version 2020.3.4 (Tableau Software, LLC, Seattle, WA). A vaccination breakthrough case was defined as a PCR-positive sample from a patient obtained $>14$ days after full vaccination (ie, both doses of the Pfizer or Moderna mRNA vaccines) was completed. For some cases, manual chart review was conducted to resolve discrepancies or ambiguities.

\section{Results}

\section{Delta Epidemiologic Wave}

The first Houston Methodist patient infected with a Delta family variant was identified in mid-April 2021, a time when the Alpha VOC was responsible for most COVID-19 cases in metropolitan Houston, and the area was experiencing a steady downturn in the total number of new COVID-19 cases (Figure 1). Delta VOCs slowly increased in frequency, but beginning in early July, a sharp uptick of COVID-19 cases caused by these VOCs occurred (Figures 1 and 2), with an estimated doubling time of approximately 7 days. By September 20, the genome data showed that Delta VOCs accounted for $99.9 \%$ of all new COVID-19 cases in our health system (Figure 2). This represents the fourth wave of COVID-19 cases in metropolitan Houston (Figure 1).

During the study period, 18,736 COVID-19 cases were diagnosed in our health care system, and 16,965 SARSCoV-2 genomes were sequenced, representing $91 \%$ of cases. A total of 13,043 patients $(76.9 \%$ of the total sequenced) were identified with Delta VOCs (Table 1). The majority [62\% (2418 of 3922)] of the non-Delta COVID-19 cases occurring during the study period were caused by the Alpha (UK B.1.1.7) VOC.

Consistent with extensive infections caused by Delta variants in Southeast Asia and elsewhere (CDC, https:// www.cdc.gov/coronavirus/2019-ncov/cases-updates/ variant-surveillance/variant-info.html, last accessed August 18, 2021; GOV.UK, https://www.gov.uk/ government/collections/new-sars-cov-2-variant, last accessed August 18, 2021), ${ }^{1}$ several patients had very recent travel histories to countries with a high prevalence of these VOCs, suggesting acquisition abroad and importation into Houston. Sixty patients with Delta Plus (Delta plus the $\mathrm{K} 417 \mathrm{~N}$ spike amino acid substitution) were identified. No cases caused by Delta variant AY.4.2 (Delta plus Y145H and A222V), a sublineage also sometimes referred to as "Delta Plus" were identified. The AY.4.2 variant has been a cause of concern because it has been reported to cause approximately $11 \%$ of new cases in the United Kingdom (Pango, https://outbreak.info/ situation-reports? pango $=A Y .4 .2 \& l o c=I N D \& l o c=G B R$ $\& l o c=U S A \&$ selected , last accessed November 4, 2021).

To understand the geospatial distribution of Delta in metropolitan Houston, patient metadata were acquired from the electronic medical record by using standard informatics methods, and home address zip codes were used to visualize virus spread (Figure 2). Delta VOCs were widely distributed throughout metropolitan Houston, with 334 distinct zip codes represented (Figure 2), indicative of the ability of the Delta variants to spread very effectively and rapidly between individuals. Analysis of Delta VOCs dissemination over time illustrated the rapid spread of these variants throughout the Houston metroplex (Figure 2). 


\section{Delta Family Subvariants}

A total of 1236 subvariants were identified based on amino acid changes in spike protein. The seven most common identified in our study are presented here (Figure 3), and these represent $73 \%$ of the 13,043 Delta samples. It is possible that the unexpectedly high number of subvariants reflects several contributing factors, including the large population sizes and genetic diversity of the Delta variant globally, as well as multiple independent introductions into the Houston area. Other factors that in principle may contribute to subvariant generation and survival include high virus load in patients on initial diagnosis (Table 1), infection of partially immunized hosts, and prolonged infections occurring in relatively immunocompromised individuals. ${ }^{10-16}$ In this regard, some of our patients had a

Table 1 Summary of Pertinent Patient Metadata for the 16,965 Unique Patients

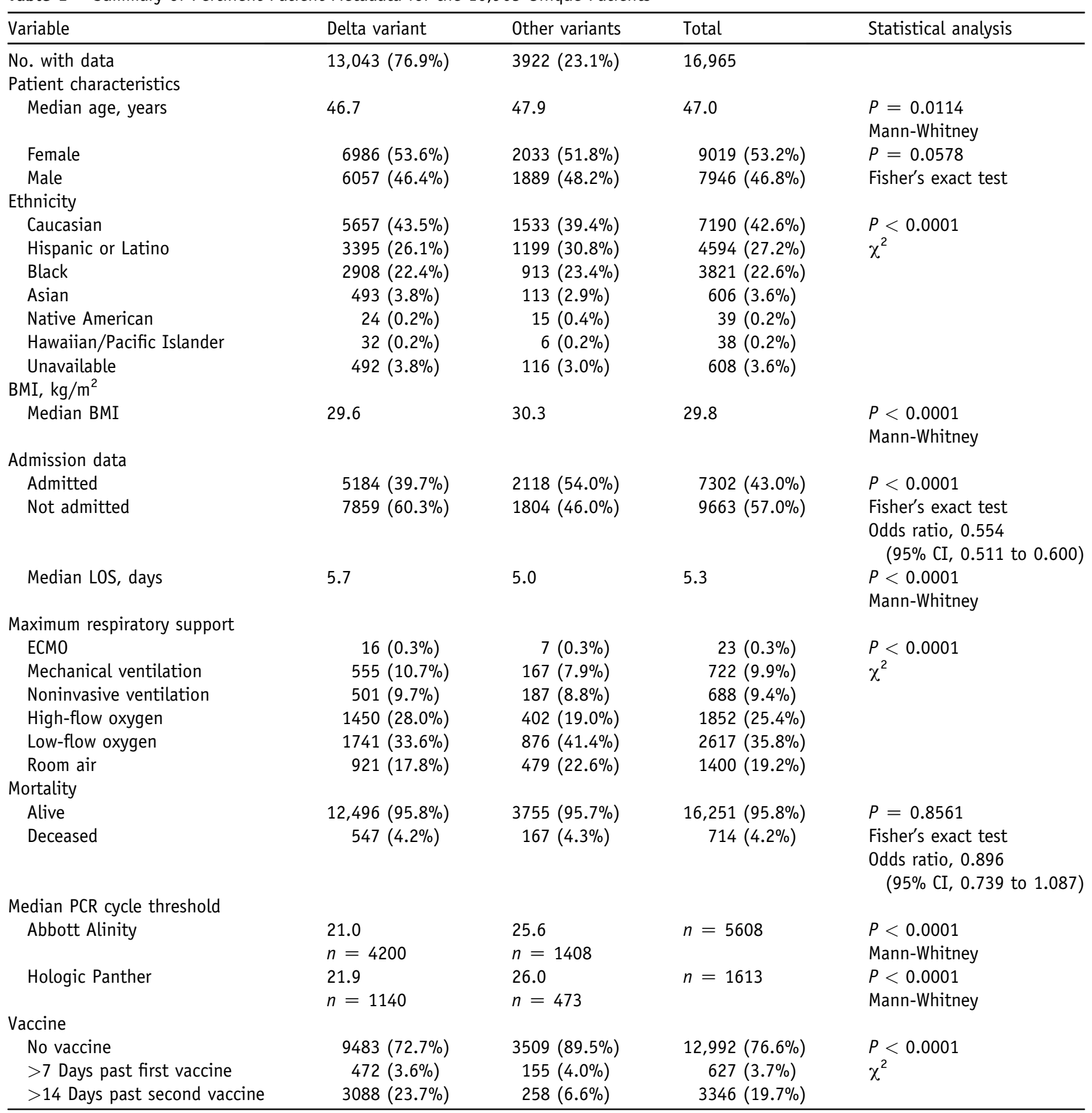

Data include 16,965 unique patients with high-quality sequence results between March 15, 2021, and September 20, 2021.

BMI, body mass index; CI, confidence interval; ECMO, extracorporeal membrane oxygenation; LOS, length of stay. 
history of cancer and organ transplants (data not shown). Patients infected with the same subvariant usually lived in different zip codes and generally had no apparent epidemiologic link, consistent with the ability of the Delta variant to spread very rapidly. In some cases, there were clear epidemiologic links between patients infected with the same subvariant, including being members of the same household.
There is a considerable lack of detailed information about patients in the United States with COVID-19 caused by Delta VOCs (World Health Organization, https://www. who.int/publications/m/item/weekly-epidemiologicalupdate-on-covid-19-13-july-2021, last accessed August 18, 2021). Compared with all other patients in the time frame studied, there was no significant difference in sex, but patients infected with Delta were

Table 2 Comparison of Pertinent Patient Metadata for 16,281 Unique Delta VOC and Alpha VOC Patients

\begin{tabular}{|c|c|c|c|c|}
\hline Variable & Delta variant & Alpha variant & Total & Statistical analysis \\
\hline No. with data & $13,043(80.1 \%)$ & $3238(19.9 \%)$ & 16,281 & \\
\hline \multicolumn{5}{|l|}{ Patient characteristics } \\
\hline Median age, years & 46.7 & 49.4 & 47.3 & $\begin{array}{l}P<0.0001 \\
\text { Mann-Whitney }\end{array}$ \\
\hline Female & $6986(53.6 \%)$ & $1698(52.4 \%)$ & $8684(53.3 \%)$ & $P=0.2538$ \\
\hline Male & $6057(46.4 \%)$ & $1540(47.6 \%)$ & $7597(46.7 \%)$ & Fisher's exact test \\
\hline \multicolumn{5}{|l|}{ Ethnicity } \\
\hline Caucasian & $5657(43.5 \%)$ & $1269(39.4 \%)$ & $6926(42.7 \%)$ & $P<0.0001$ \\
\hline Hispanic or Latino & $3395(26.1 \%)$ & $961(29.8 \%)$ & $4356(26.9 \%)$ & $\chi^{2}$ \\
\hline Black & $2908(22.4 \%)$ & $757(23.5 \%)$ & $3665(22.6 \%)$ & \\
\hline Asian & $493(3.8 \%)$ & $126(3.9 \%)$ & $619(3.8 \%)$ & \\
\hline Native American & $24(0.2 \%)$ & $12(0.4 \%)$ & $36(0.2 \%)$ & \\
\hline Hawaiian/Pacific Islander & $32(0.2 \%)$ & $4(0.1 \%)$ & $36(0.2 \%)$ & \\
\hline Unavailable & $492(3.8 \%)$ & $91(2.8 \%)$ & $583(3.6 \%)$ & \\
\hline \multicolumn{5}{|l|}{ BMI, $\mathrm{kg} / \mathrm{m}^{2}$} \\
\hline Median BMI & 29.6 & 30.5 & 29.8 & $\begin{array}{l}P<0.0001 \\
\text { Mann-Whitney }\end{array}$ \\
\hline \multicolumn{5}{|l|}{ Admission data } \\
\hline Admitted & $5184(39.7 \%)$ & $1721(53.2 \%)$ & $6905(42.4 \%)$ & $P<0.0001$ \\
\hline Not admitted & $7859(60.3 \%)$ & $1517(46.8 \%)$ & $9376(57.6 \%)$ & $\begin{array}{l}\text { Fisher's exact test } \\
\text { Odds ratio, } 0.581 \\
\quad(95 \% \text { CI, } 0.538 \text { to } 0.628)\end{array}$ \\
\hline Median LOS, days & 5.7 & 5.1 & 5.4 & $\begin{array}{l}P=0.0031 \\
\text { Mann-Whitney }\end{array}$ \\
\hline \multicolumn{5}{|l|}{ Maximum respiratory support } \\
\hline ECMO & $16(0.3 \%)$ & $7(0.4 \%)$ & $23(0.3 \%)$ & $P<0.0001$ \\
\hline Mechanical ventilation & $555(10.7 \%)$ & $155(9.0 \%)$ & $710(10.3 \%)$ & $\chi^{2}$ \\
\hline Noninvasive ventilation & $501(9.7 \%)$ & $165(9.6 \%)$ & $666(9.6 \%)$ & \\
\hline High-flow oxygen & $1450(28.0 \%)$ & $366(21.3 \%)$ & $1816(26.3 \%)$ & \\
\hline Low-flow oxygen & $1741(33.6 \%)$ & $705(41.0 \%)$ & $2446(35.4 \%)$ & \\
\hline Room air & $921(17.8 \%)$ & $323(18.8 \%)$ & $1244(18.0 \%)$ & \\
\hline \multicolumn{5}{|l|}{ Mortality } \\
\hline Alive & $12,496(95.8 \%)$ & $3073(94.9 \%)$ & $15,569(95.6 \%)$ & $P=0.0271$ \\
\hline Deceased & $547(4.2 \%)$ & $165(5.1 \%)$ & $712(4.4 \%)$ & $\begin{array}{l}\text { Fisher's exact test } \\
\text { Odds ratio, } 0.815 \\
\quad(95 \% \text { CI, } 0.682 \text { to } 0.975)\end{array}$ \\
\hline \multicolumn{5}{|l|}{ Median PCR cycle threshold } \\
\hline Abbott Alinity & $\begin{array}{l}21.0 \\
n=4200\end{array}$ & $\begin{array}{l}22.4 \\
n=1101\end{array}$ & $n=5301$ & $\begin{array}{l}P=0.0004 \\
\text { Mann-Whitney }\end{array}$ \\
\hline Hologic Panther & $\begin{array}{l}21.9 \\
n=1140\end{array}$ & $\begin{array}{l}24.3 \\
n=374\end{array}$ & $n=1514$ & $\begin{array}{l}P<0.0001 \\
\text { Mann-Whitney }\end{array}$ \\
\hline \multicolumn{5}{|l|}{ Vaccine } \\
\hline No vaccine & $9483(72.7 \%)$ & $2969(91.7 \%)$ & $12,452(76.5 \%)$ & $P<0.0001$ \\
\hline$>7$ Days past first vaccine & $472(3.6 \%)$ & $133(4.1 \%)$ & $605(3.7 \%)$ & $\chi^{2}$ \\
\hline$>14$ Days past second vaccine & $3088(23.7 \%)$ & $136(4.2 \%)$ & $3224(19.8 \%)$ & \\
\hline
\end{tabular}

Data include 16,281 unique patients with high-quality sequence results between January 1, 2021, and September 20, 2021.

BMI, body mass index; CI, confidence interval; ECMO, extracorporeal membrane oxygenation; LOS, length of stay. 
Table 3 Summary of Pertinent Patient Metadata for the 3346 Fully Vaccinated Patients

\begin{tabular}{|c|c|c|c|c|}
\hline Variable & Vaccinated & Not vaccinated & Total & Statistical analysis \\
\hline \multicolumn{5}{|c|}{ Median PCR cycle threshold } \\
\hline Abbott Alinity & $\begin{array}{l}20.5 \\
n=1244\end{array}$ & $\begin{array}{l}22.1 \\
n=4364\end{array}$ & $n=5608$ & $\begin{array}{l}P=0.0018 \\
\text { Mann-Whitney }\end{array}$ \\
\hline Hologic Panther & $\begin{array}{l}22.2 \\
n=378\end{array}$ & $\begin{array}{l}23.5 \\
n=1235\end{array}$ & $n=1613$ & $\begin{array}{l}P=0.0348 \\
\text { Mann-Whitney }\end{array}$ \\
\hline \multicolumn{5}{|l|}{ Admission data } \\
\hline Not admitted & Delta variant & Other variants & Total & \\
\hline $\begin{array}{l}\text { No. with data } \\
\text { Admission data }\end{array}$ & $3088(92.3 \%)$ & $258(7.7 \%)$ & 3346 & \\
\hline
\end{tabular}

significantly but modestly younger (Table 1). However, Delta variant patients were admitted significantly less frequently than other patients and had a significantly longer median hospital length of stay; there was no significant difference in mortality rate (Table 1). Delta cases were more likely to be Asian (consistent with multiple recent entry points from abroad) and Caucasian, and less likely to be Hispanic or Latino, and to have

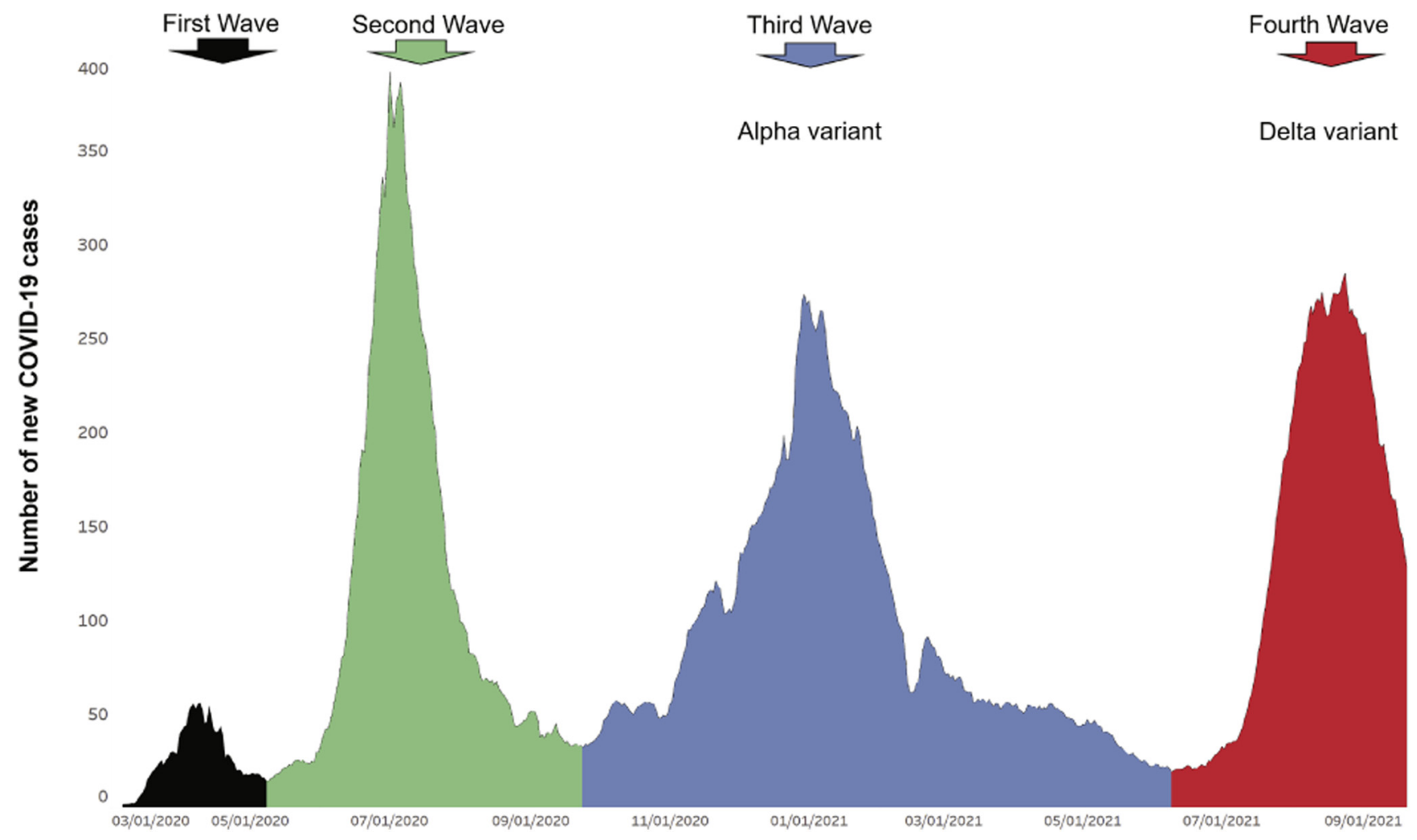

Figure 1 Epidemiologic curve showing four coronavirus disease 2019 (COVID-19) waves in Houston Methodist patients. Number of new COVID-19 cases (y axis) totals are shown as a \pm 3 -day moving average. Each of the four waves is shown in a different color simply as a stylistic device. The first and second waves were composed of a heterogeneous array of severe acute respiratory syndrome coronavirus 2 genotypes. The Alpha variant shown in the third wave and the Delta variant shown in the fourth wave indicate their numeric prominence in those waves and should not be interpreted to mean that all cases in the third and fourth waves were caused by Alpha and Delta variants of concern, respectively. The fourth wave shown includes data through September 20, 2021. The figure was generated with Tableau version 2020.3.4 (Tableau Software, LLC). The curve mirrors that of disease activity in metropolitan Houston, Texas. 


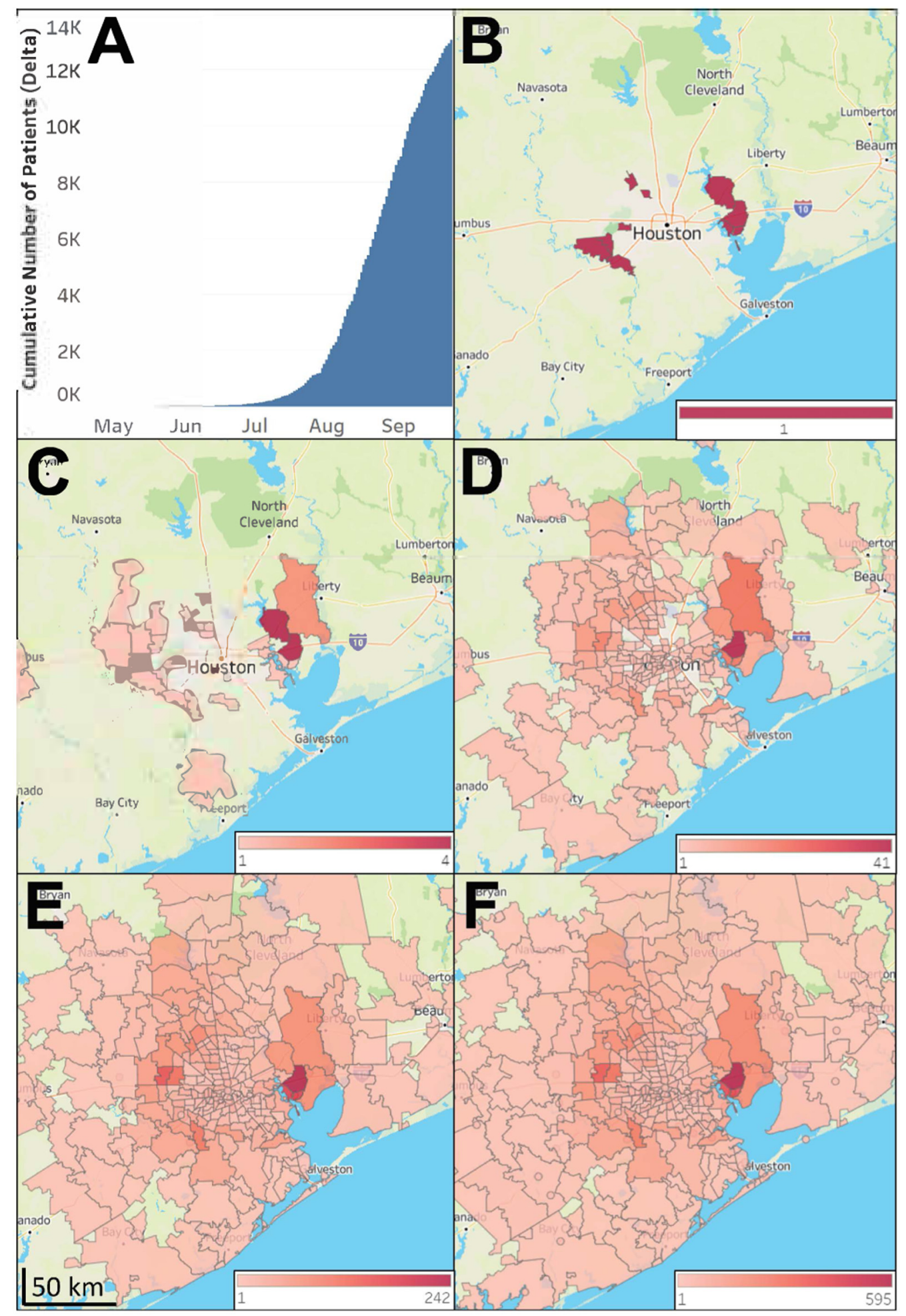

Figure 2 Increase in Delta frequency over time and distribution in metropolitan Houston. The study time frame was March 15, 2021, through September 20, 2021. A: Cumulative increase in Delta during the study period; $y$ axis is the cumulative number of new coronavirus disease 2019 cases. At the end of the study period, Delta continuously caused $99.9 \%$ of all cases of coronavirus disease 2019. B-E: Geospatial distribution of Delta based on home address zip code for each patient. B: April 15 to May 15. C: April 15 to June 15. D: April 15 to July 15. E: April 15 to August 15. F: April 15 to September 20. Note differences in heat map scale for each panel. Figures were generated by using Tableau version 2020.3.4.

significantly lower PCR cycle threshold values on initial diagnosis (a proxy for higher virus load, a finding consistent with increased transmissibility of Delta variants). In addition, Delta variants caused a significantly higher rate of vaccine breakthrough cases $(23.7 \%$ compared with $6.6 \%$ for all other variants combined) 
S1-NTD

S1-RBD

S1

S2

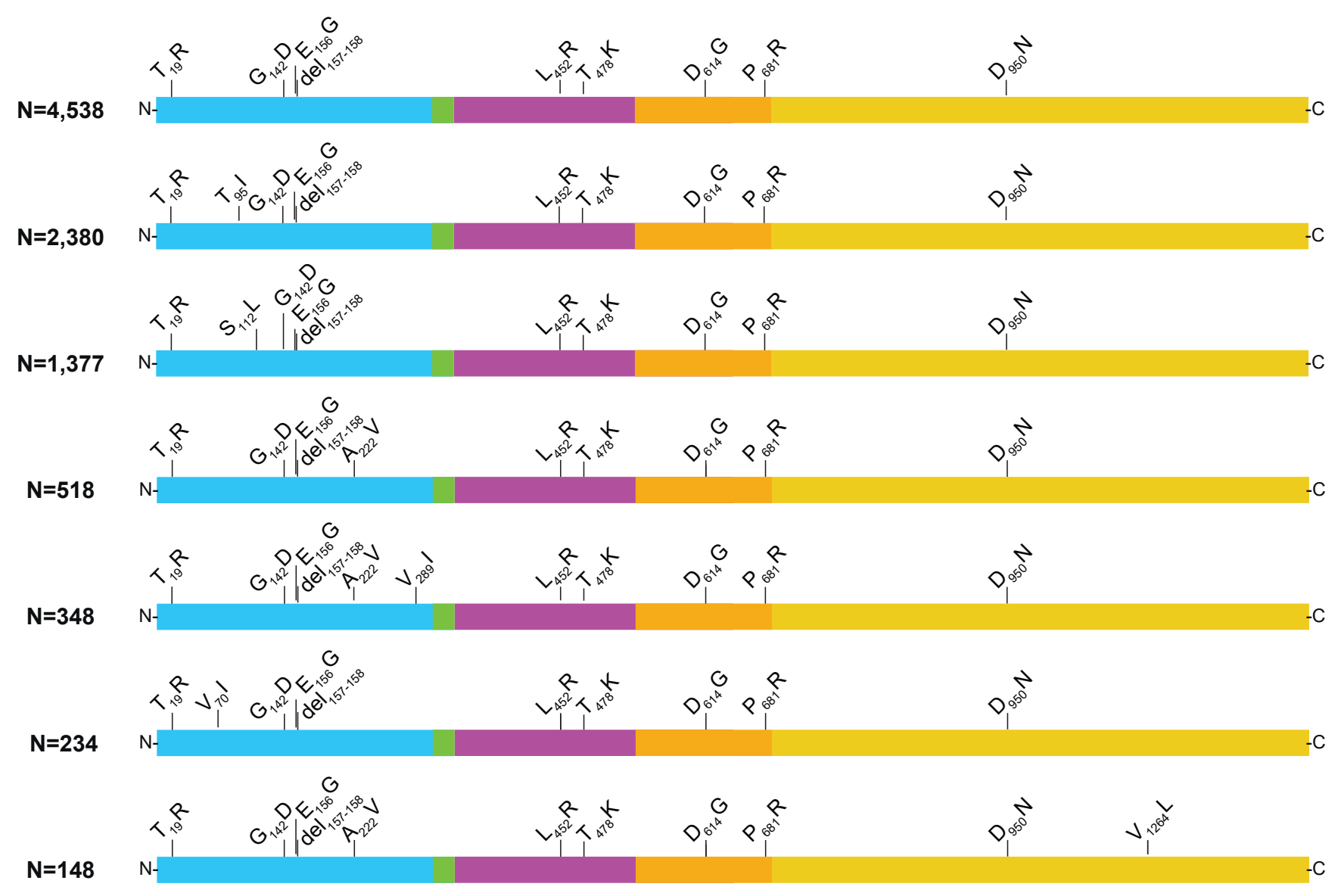

$\mathrm{N}=\mathbf{2 , 3 8 0}$

Figure 3 Structural changes present in spike protein of the seven most abundant Delta subvariants identified in the study. The numbers denote the number of patients with each subvariant identified in this study. These seven subvariants represent $73 \%$ of the 13,043 Delta samples identified in the study. The figure includes all subvariants represented by at least 100 samples. Data presented in this image build on information that has been previously published. ${ }^{7,8}$ S1, $\mathrm{S} 1$ domain; S2, S2 domain; S1-NTD, S1 domain-aminoterminal domain; S1-RBD, S1 domain-receptor binding domain.

(Table 1). Consistent with Delta causing an increased number of vaccine breakthrough cases, this variant reportedly has reduced sensitivity to antibody neutralization in vitro. ${ }^{14}$

\section{Comparison of Delta and Alpha COVID-19 Cases}

The occurrence of a prominent third wave of COVID-19 cases in Houston caused by the Alpha VOC (Figure 1) provided the opportunity to compare and contrast large disease waves caused by two genetically distinct SARSCoV-2 genotypes. For this analysis, Alpha VOC cases were included beginning January 1, 2021, because that time was soon after the first recorded Houston Methodist case caused by this variant. The number of Delta cases studied ( $n=13,043$ ) was much greater than the number of Alpha cases $(n=3238)$ (Table 2$)$. The two patient populations differed significantly in many characteristics, including median age, ethnicity, median PCR cycle threshold level, admission rates, maximum respiratory support, rate of vaccine breakthrough, median length of stay, and mortality (Table 2).

\section{Vaccine Breakthrough Cases}

Vaccine breakthrough cases were analyzed next (Table 3). Of the 16,965 total patients, 3346 (19.7\%) patients, for whom genome sequence data were available, met the CDC definition of vaccine breakthrough cases (Tables 1 and 3). There was no simple relationship between the time elapsed since administration of the second booster vaccination and the date of vaccination breakthrough (data not shown). These 3346 patients received either the Pfizer-BioNTech BNT162b2 $[n=2829(85 \%)]$, Moderna mRNA-1273 $[n=365(11 \%)]$, or J\&J/Janssen JNJ-78436735 $[n=147(4 \%)]$ vaccine; vaccine type was not specified for five individuals. This distribution generally reflects the great majority of BNT162b2 vaccination doses given in our health system and should not be interpreted to mean that the Pfizer-BioNTech and Moderna products are unusually prone 
to breakthrough cases, as these mRNA vaccines are highly efficacious. ${ }^{6,17-23}$ Vaccinated patients infected with nonDelta variants had a significantly higher cycle threshold value on initial diagnosis, likely indicating better vaccine protection, lower virus load, and decreased probability of transmission. Compared with all other variants combined, a significantly lower percentage $(25.9 \%$ versus $37.2 \%$; $P=0.0001$ ) of patients with breakthrough cases caused by Delta variants were admitted to the hospital (Tables 1 and $3)$.

\section{Lambda and Mu Variants of Interest}

The Lambda variant of interest was first reported in November 2020. It has very recently attracted widespread media and public interest because it has caused large numbers of infections in Peru and Ecuador and has been reported to cause COVID-19 in the United States ${ }^{24,25}$ (World Health Organization, https://www.who.int/en/ activities/tracking-SARS-CoV-2-variants/, last accessed September 2, 2021). There has been speculation that Lambda may become numerically prominent in the United States and other countries, in part because its spike protein differs immunologically and has been reported to be partially resistant in vitro to vaccine-induced sera and therapeutic monoclonal antibodies. ${ }^{26-28}$ Because of this concern, our large genome sequence database was inspected to determine if Lambda was present, and if so, if it had increased substantially in the Houston metropolitan region. The analysis identified only eight cases caused by this VOI, with the first one identified in our Houston Methodist patient population in mid-July 2021 (Figure 4A). These patients lived in several noncontiguous zip codes throughout the metroplex; that is, they were not restricted to a single geographic area of greater Houston (Figure 4, A and B). The $\mathrm{Mu}$ VOI was initially identified in Colombia in January 2021 , and has been reported to account for $39 \%$ of cases in that country. ${ }^{29} \mathrm{Mu}$ also has been found in many other countries worldwide, and recently it was reported that the $\mathrm{Mu}$ variant has increased resistance in vitro to antibodies elicited by vaccination with BNT162b2 and natural infection by SARS-CoV-2. ${ }^{30}$ Fifty three cases of COVID-19 caused by the Mu VOI were identified. Similar to the Lambda, these cases were distributed in multiple areas in the Houston metropolitan region (Figure 4, C and D).

\section{Discussion}

This work analyzed SARS-CoV-2 Delta VOC population genomics and patient characteristics for 16,965 patients, focusing on mid-March 2021 through late September 2021, a time frame in which there was total replacement of the previously dominant Alpha (B.1.1.7) VOC by Delta family VOCs. During this 6-month period, a substantial increase in COVID-19 cases occurred in our health care system and throughout all of the Houston metropolitan area, virtually all driven by rapid dissemination of the highly contagious Delta family variants. The study was based predominantly on genome sequence analysis of 16,965 SARS-CoV-2 samples from socioeconomically, geographically, and ethnically diverse patients. Several key findings were made, including: i) Delta family VOCs supplanted the Alpha VOC in a relatively short period of time, ii) regardless of vaccination status, on initial diagnosis, compared with patients infected by other VOCs, patients infected by Delta VOCs had significantly lower cycle threshold values, likely indicating significantly higher viral load in the nasopharynx, iii) Delta caused significantly more vaccine breakthrough cases than other VOCs, iv) significantly fewer fully vaccinated individuals required hospitalization, and v) the Lambda and $\mathrm{Mu}$ VOIs were identified but were very rare, and they did not increase to substantial levels in the time frame studied.

The current genome sequence data document a rapid increase in Delta variant cases in metropolitan Houston and a corresponding decrease in COVID-19 cases caused by Alpha and other variants, findings similar to epidemiologic trends observed in the United Kingdom (World Health Organization, https://www.who.int/publications $/ \mathrm{m} / \mathrm{item} /$ weekly-epidemiological-update-on-covid-19-13-july-2021, last accessed August 18, 2021; Office for National Statistics, https://www.ons.gov.uk/peoplepopulationandcommunity/ healthandsocialcare/conditionsanddiseases/bulletins/ coronaviruscovid19infectionsurveypilot/9july2021, last accessed August 18, 2021). In $<5$ months, Delta VOCs increased from an initial documented case in our large patient population to cause $99.9 \%$ of all cases in our health care system. The rapid increase in Delta cases documented is responsible for a prominent fourth wave of COVID-19 in Houston that is ongoing (Figure 1). The current findings are consistent with Delta VOC epidemiologic data reported from other regions of the United States.

Delta was significantly more likely to cause vaccine breakthrough cases (Tables 1 and 3). However, importantly, $19.7 \%$ of all the 16,965 COVID-19 cases with genome sequence data occurred in fully vaccinated individuals. Importantly, only a small number $[n=896(5.3 \%)]$ of these patients required hospitalization. Vaccine breakthrough cases have emerged as an area of great interest, especially so with the increasing percentage of COVID-19 cases caused by Delta variants and the recognition that they are important causes of breakthroughs. ${ }^{31-36}$ Although the current analysis did not identify a simple relationship between the time elapsed since administration of the second booster vaccination and the date of vaccination breakthrough, this is an important area for continued study. Although the potential relationship between vaccination breakthrough and waning immunity were not studied, studies of this topic are ongoing.

Some investigators have speculated that the Lambda and $\mathrm{Mu}$ variants may become a major concern in future surges. Although in principle this is possible, the current data show 


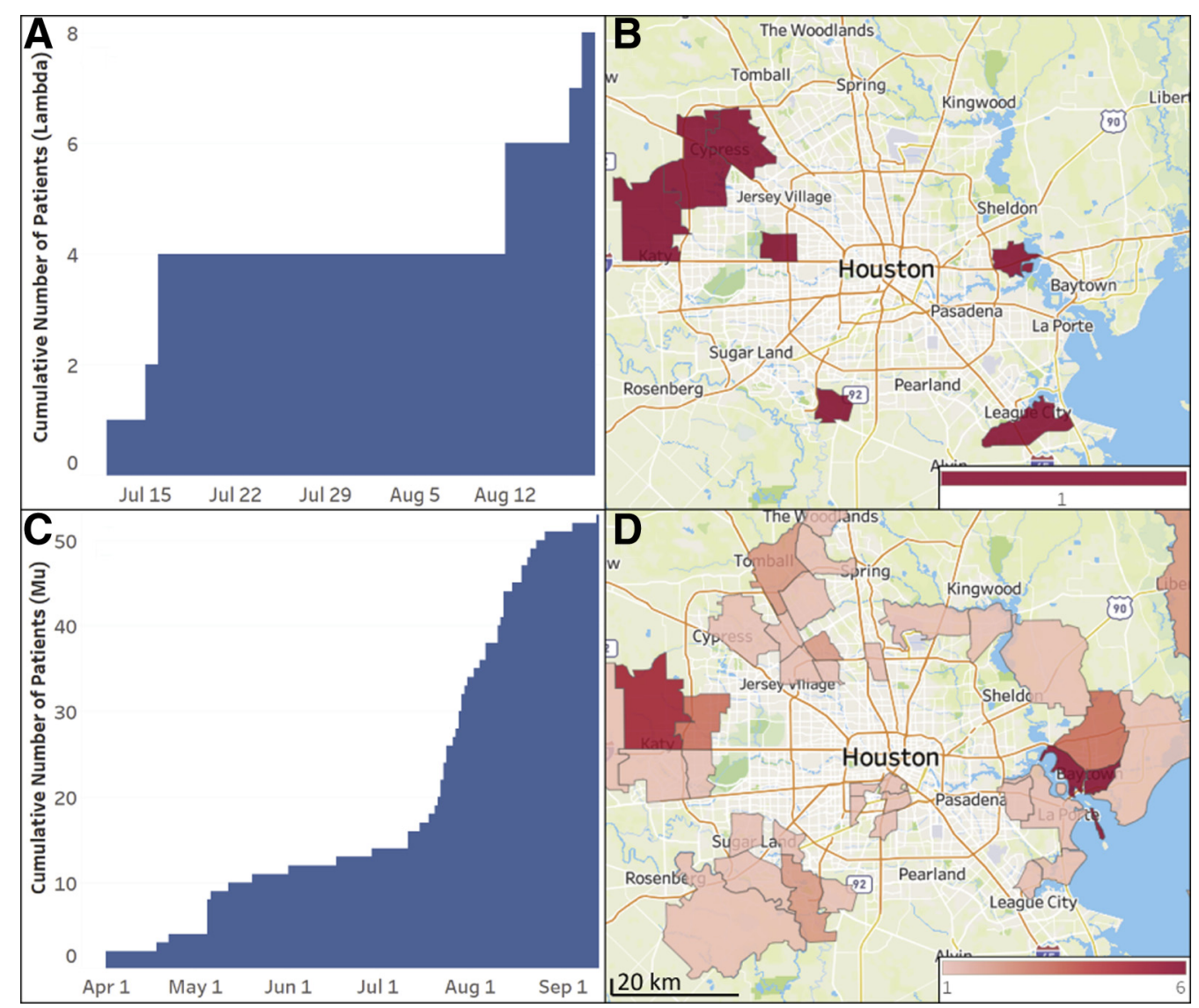

Figure 4 Lambda and Mu variants identified in metropolitan Houston in our patient population. A: Cumulative increase in the cases caused by Lambda variants identified in the study. B: Geospatial distribution of these Lambda variants based on the home address zip code for each patient. C: Cumulative increase in cases caused by the Mu variants identified in the study. D: Geospatial distribution of the Mu variants based on the home address zip code for each patient. Figures were generated by using Tableau version 2020.3.4.

that neither variant has increased substantially in our metropolitan area during the time frame studied. Thus, the genome analysis of a large set of samples from the first 9 months of 2021 does not currently support this speculation, although circumstances may change in the future. Because a great majority of SARS-CoV-2 genomes causing COVID19 were sequenced in our diverse patient population, the growth trajectory of these and other variants in a major metropolitan region in the United States were continuously monitored. Thus, the ongoing near-real-time sequencing of SARS-CoV-2 genomes responsible for COVID-19 cases in metropolitan Houston provides a facile strategy to assess changes in the virus population composition and molecular evolution in this populous area.

The current study has several limitations that should be noted. Although the genomes of SARS-CoV-2 causing 91\% of all Houston Methodist COVID-19 cases in the study period were sequenced, this sample represents only approximately $5 \%$ of cases reported in the metropolitan region. It is possible that our patient populations underrepresent certain demographic groups, including homeless individuals and pediatric patients. The great majority of the samples sequenced in this study were obtained from symptomatic patients. Thus, it is possible that our sample failed to identify virus genotypes that are preferentially represented in asymptomatic individuals. However, given that at the end of the study Delta represented $99.9 \%$ of all cases, it is unlikely that variants associated with asymptomatic individuals would be substantially different, although this is a formal possibility.

In the aggregate, the current data add critical new information to the finding that these three vaccines are highly efficacious in decreasing severe COVID-19, hospitalizations, and deaths. ${ }^{6,20,23}$ Furthermore, the current study highlights the importance of analyzing SARS-CoV-2 genome data integrated with linked patient metadata and stresses the need to continue to do this in near real time as the pandemic continues, the virus evolves, and new variants with potentially increased fitness are generated. Analyses of this type are also important in the context of vaccine formulation and long COVID, an increasing health and economic problem globally.

\section{Acknowledgments}

We thank Drs. Marc Boom and Dirk Sostman for their ongoing support, Dr. Sasha Pejerrey for editorial 
contributions, and Dr. Heather McConnell for help with figures. The research was supported by the Houston Methodist Academic Institute Infectious Diseases Fund and many generous Houston philanthropists. The funders had no role in the design and conduct of the study; collection, management, analysis, and interpretation of the data; preparation, review, or approval of the manuscript; or decision to submit the manuscript for publication.

\section{Author Contributions}

P.A.C., R.J.O., S.W.L., S.S., and J.M.M. had full access to all study data and take responsibility for the integrity of the data and the accuracy of the data analysis; J.M.M., P.A.C., R.J.O., and S.W.L. conceived the study; P.A.C. performed statistical analysis; J.M.M. and J.J.D. obtained funding; and J.M.M. supervised the study. All authors contributed to data acquisition, analysis, or interpretation, and all authors drafted the manuscript.

\section{Supplemental Data}

Supplemental material for this article can be found at http://doi.org/10.1016/j.ajpath.2021.10.019.

\section{References}

1. Dhar MS, Marwal R, Radhakrishnan V, Ponnusamy K, Jolly B, Bhoyar RC, et al: Genomic characterization and epidemiology of an emerging SARS-CoV-2 variant in Delhi, India. medRxiv 2021, [Preprint] doi: 10.1101/2021.06.02.21258076

2. Hacisuleyman E, Hale C, Saito Y, Blachere NE, Bergh M, Conlon EG, Schaefer-Babajew DJ, DaSilva J, Muecksch F, Gaebler C, Lifton R, Nussenzweig MC, Hatziioannou T, Bieniasz PD, Darnell RB: Vaccine breakthrough infections with SARS-CoV-2 variants. N Engl J Med 2021, 384:2212-2218

3. Brosh-Nissimov T, Orenbuch-Harroch E, Chowers M, Elbaz M, Nesher L, Stein M, Maor Y, Cohen R, Hussein K, Weinberger M, Zimhony O, Chazan B, Najjar R, Zayyad H, Rahav G, WienerWell Y: BNT162b2 vaccine breakthrough: clinical characteristics of 152 fully-vaccinated hospitalized COVID-19 patients in Israel. Clin Microbiol Infect 2021, 27:1652-1657

4. CDC COVID-19 Vaccine Breakthrough Case Investigations Team, Birhane M, Bressler, et al: COVID-19 vaccine breakthrough infections reported to CDC-United States, January 1-April 30, 2021. MMWR Morb Mortal Wkly Rep 2021, 70:792-793

5. Kustin T, Harel N, Finkel U, Perchik S, Harari S, Tahor M, Caspi I, Levy R, Leshchinsky M, Dror SK, Bergerzon G, Gadban H, Gadban F, Eliassian E, Shimron O, Saleh L, Ben-Zvi H, Taraday EK, Amichay D, Ben-Dor A, Sagas D, Strauss M, Shemer Avni Y, Huppert A, Kepten E, Balicer RD, Netzer D, Ben-Shachar S, Stern A: Evidence for increased breakthrough rates of SARS-CoV-2 variants of concern in BNT162b2-mRNA-vaccinated individuals. Nat Med 2021, 27:1379-1384

6. Tenforde MW, Patel MM, Ginde AA, Douin DJ, Talbot HK, Casey JD, et al: Effectiveness of severe acute respiratory syndrome coronavirus 2 messenger RNA vaccines for preventing coronavirus disease 2019 hospitalizations in the United States. Clin Infect Dis 2021, [Epub ahead of print] doi: 10.1093/cid/ciab687

7. Long SW, Olsen RJ, Christensen PA, Subedi S, Olson R, Davis JJ, Saavedra MO, Yerramilli P, Pruitt L, Reppond K, Shyer MN,
Cambric J, Finkelstein IJ, Gollihar J, Musser JM: Sequence analysis of 20,453 severe acute respiratory syndrome coronavirus 2 genomes from the Houston metropolitan area identifies the emergence and widespread distribution of multiple isolates of all major variants of concern. Am J Pathol 2021, 191:983-992

8. Olsen RJ, Christensen PA, Long SW, Subedi S, Hodjat P, Olson R, Nguyen M, Davis JJ, Yerramilli P, Saavedra MO, Pruitt L, Reppond K, Shyer MN, Cambric J, Gadd R, Thakur RM, Batajoo A, Finkelstein IJ, Gollihar J, Musser JM: Trajectory of growth of severe acute respiratory (SARS-CoV-2) syndrome coronavirus 2 variants in Houston, Texas, January through May 2021, based on 12,476 genome sequences. Am J Pathol 2021, 191:1754-1773

9. Davis JJ, Long SW, Christensen PA, Olsen RJ, Olson R, Shukla M, Subedi S, Stevens R, Musser JM: Analysis of the ARTIC version 3 and version 4 SARS-CoV-2 primers and their impact on the detection of the G142D amino acid substitution in the spike protein. bioRxiv 2021, [Preprint] doi: 10.1101/2021.09.27.461949

10. Clark SA, Clark LE, Pan J, Coscia A, McKay LGA, Shankar S, Johnson RI, Brusic V, Choudhary MC, Regan J, Li JZ, Griffiths A, Abraham J: SARS-CoV-2 evolution in an immunocompromised host reveals shared neutralization escape mechanisms. Cell 2021, 184 2605-2617.e18

11. Choi B, Choudhary MC, Regan J, Sparks JA, Padera RF, Qiu X, Solomon IH, Kuo H-H, Boucau J, Bowman K, Adhikari UD Winkler ML, Mueller AA, Hsu TY-T, Desjardins M, Baden LR, Chan BT, Walker BD, Lichterfeld M, Brigl M, Kwon DS, Kanjilal S, Richardson ET, Jonsson AH, Alter G, Barczak AK, Hanage WP, Yu XG, Gaiha GD, Seaman MS, Cernadas M, Li JZ: Persistence and evolution of SARS-CoV-2 in an immunocompromised host. N Engl J Med 2020, 383:2291-2293

12. Baang JH, Smith C, Mirabelli C, Valesano AL, Manthei DM, Bachman MA, Wobus CE, Adams M, Washer L, Martin ET, Lauring AS: Prolonged severe acute respiratory syndrome coronavirus 2 replication in an immunocompromised patient. J Infect Dis 2021, 223:23-27

13. Hensley MK, Bain WG, Jacobs J, Nambulli S, Parikh U, Cillo A, Staines B, Heaps A, Sobolewski MD, Rennick LJ, Macatangay BJC, Klamar-Blain C, Kitsios GD, Methé B, Somasundaram A, Bruno TC, Cardello C, Shan F, Workman C, Ray P, Ray A, Lee J, Sethi R, Schwarzmann WE, Ladinsky MS, Bjorkman PJ, Vignali DA, Duprex WP, Agha ME, Mellors JW, McCormick KD, Morris A, Haidar G: Intractable coronavirus disease 2019 (COVID-19) and prolonged severe acute respiratory syndrome coronavirus 2 (SARS$\mathrm{CoV}-2)$ replication in a chimeric antigen receptor-modified T-cell therapy recipient: a case study. Clin Infect Dis 2021, 73:e815-e821

14. Planas D, Veyer D, Baidaliuk A, Staropoli I, Guivel-Benhassine F, Rajah MM, Planchais C, Porrot F, Robillard N, Puech J, Prot M, Gallais F, Gantner P, Velay A, Le Guen J, Kassis-Chikhani N, Edriss D, Belec L, Seve A, Courtellemont L, Péré H, Hocqueloux L, Fafi-Kremer S, Prazuck T, Mouquet H, Bruel T, Simon-Lorière E, Rey FA, Schwartz O: Reduced sensitivity of SARS-CoV-2 variant Delta to antibody neutralization. Nature 2021, 596:276-280

15. Kemp SA, Collier DA, Datir RP, Ferreira IATM, Gayed S, Jahun A: SARS-CoV-2 evolution during treatment of chronic infection. Nature 2021, 592:277-282

16. Nussenblatt V, Roder AE, Das S, de Wit E, Youn J-H, Banakis S, Muchegian A, Mederos C, Wang W, Chung M, Perez-Perez L, Palmore T, Brudno J, Kochenderfer J, Ghedin E: Year-long COVID19 infection reveals within-host evolution of SARS-CoV-2 in a patient with B cell depletion. medRxiv 2021, [Preprint] doi: 10.1101/ 2021.10.02.21264267

17. Polack FP, Thomas SJ, Kitchin N, Absalon J, Gurtman A, Lockhart S, Perez JL, Pérez Marc G, Moreira ED, Zerbini C, Bailey R, Swanson KA, Roychoudhury S, Koury K, Li P, Kalina WV, Cooper D, Frenck RW Jr, Hammitt LL, Türeci Ö, Nell H, Schaefer A, Ünal S, Tresnan DB, Mather S, Dormitzer PR, Şahin U, Jansen KU, Gruber WC; C4591001 Clinical Trial Group: Safety and 
efficacy of the BNT162b2 mRNA Covid-19 vaccine. N Engl J Med 2020, 383:2603-2615

18. Baden LR, El Sahly HM, Essink B, Kotloff K, Frey S, Novak R, et al: Efficacy and safety of the mRNA-1273 SARS-CoV-2 vaccine. N Engl J Med 2020, 384:403-416

19. Voysey M, Clemens SAC, Madhi SA, Weckx LY, Folegatti PM, Aley PK, et al: Safety and efficacy of the ChAdOx $1 \mathrm{nCoV}-19$ vaccine (AZD1222) against SARS-CoV-2: an interim analysis of four randomised controlled trials in Brazil, South Africa, and the UK. Lancet 2021, 397:99-111

20. Lopez Bernal J, Andrews N, Gower C, Gallagher E, Simmons R, Thelwall S, Stowe J, Tessier E, Groves N, Dabrera G, Myers R, Campbell CNJ, Amirthalingam G, Edmunds M, Zambon M, Brown KE, Hopkins S, Chand M, Ramsay M: Effectiveness of Covid-19 vaccines against the B.1.617.2 (Delta) variant. N Engl J Med 2021, 385:585-594

21. Abu-Raddad LJ, Chemaitelly H, Butt AA; National Study Group for COVID-19 Vaccination: Effectiveness of the BNT162b2 Covid-19 vaccine against the B.1.1.7 and B.1.351 variants. N Engl J Med 2021, 385:187-189

22. Dagan N, Barda N, Kepten E, Miron O, Perchik S, Katz MA, Hernán MA, Lipsitch M, Reis B, Balicer RD: BNT162b2 mRNA Covid-19 vaccine in a nationwide mass vaccination setting. N Engl J Med 2021, 384:1412-1423

23. Jagadeesh Kumar V, Sowpati DT, Munigela A, Banu S, Siva AB, Sasikala M, Nutalapati C, Kulkarni A, Mukherjee P, Zaveri L; CCMB COVID-19 Team; AIG Hospitals COVID-19 Vaccine study Team, Tallapaka KB, Reddy DN: Clinical outcomes in vaccinated individuals hospitalized with Delta variant of SARS-CoV-2. medRxiv 2021, [Preprint] doi: 10.1101/2021.07.13.21260417

24. Padilla-Rojas C, Jimenez-Vasquez V, Hurtado V, Mestanza O, Molina IS, Barcena L, Morales Ruiz S, Acedo S, Lizarraga W, Bailon H, Cáceres O, Galarza M, Rojas-Serrano N, Vargas-Herrera N, Lope-Pari P, Huayra J, Solari L: Genomic analysis reveals a rapid spread and predominance of lambda (C.37) SARS-COV-2 lineage in Peru despite circulation of variants of concern. J Med Virol 2021, 93 : 6845-6849

25. Romero PE, Dávila-Barclay A, Salvatierra G, González L, Cuicapuza D, Solis L, Marcos-Carbajal P, Huancachoque J, Maturrano L, Tsukayama P: The emergence of SARS-CoV-2 variant lambda (C.37) in South America. medRxiv 2021, [Preprint] doi: 10. 1101/2021.06.26.21259487

26. Tada T, Zhou H, Dcosta BM, Samanovic MI, Mulligan MJ, Landau NR: The spike proteins of SARS-CoV-2 B.1.617 and B.1.618 variants identified in India provide partial resistance to vaccineelicited and therapeutic monoclonal antibodies. bioRxiv 2021, [Preprint] doi: 10.1101/2021.05.14.444076

27. Kimura I, Kosugi Y, Wu J, Yamasoba D, Butlertanaka EP, Tanaka YL, Liu Y, Shirakawa K, Kazuma Y, Nomura R, Horisawa Y, Tokunaga K, Takaori-Kondo A, Arase H; The Genotype to Phenotype Japan (G2P-Japan) Consortium, Saito A, Nakagawa S, Sato K: SARS-CoV-2 Lambda variant exhibits higher infectivity and immune resistance. bioRxiv 2021, [Preprint] doi: 10.1101/2021.07. 28.454085

28. Liu H, Wei P, Zhang Q, Aviszus K, Linderberger J, Yang J, Liu J, Chen Z, Waheed H, Reynoso L, Downey GP, Frankel SK, Kappler J, Marrack P, Zhang G: The Lambda variant of SARS-CoV-2 has a better chance than the Delta variant to escape vaccines. bioRxiv 2021, [Preprint] doi: 10.1101/2021.08.25.457692

29. Laiton-Donato K, Franco-Muñoz C, Álvarez-Díaz DA, RuizMoreno HA, Usme-Ciro JA, Prada DA, Reales-González J, Corchuelo S, Herrera-Sepúlveda MT, Naizaque J, Santamaría G, Rivera J, Rojas P, Ortiz JH, Cardona A, Malo D, Prieto-Alvarado F, Gómez FR, Wiesner M, Martínez MLO, Mercado-Reyes M: Characterization of the emerging B.1.621 variant of interest of SARSCoV-2. Infect Genet Evol 2021, 95:105038

30. Uriu K, Kimura I, Shirakawa K, Takaori-Kondo A, Nakada T-a, Kaneda A; The Genotype to Phenotype Japan (G2P-Japan) Consortium, Nakagawa S, Sato K: Ineffective neutralization of the SARSCoV-2 Mu variant by convalescent and vaccine sera. bioRxiv 2021, [Preprint] doi: 10.1101/2021.09.06.459005

31. Brown CM, Vostok J, Johnson H, Burns M, Gharpure R, Sami S, Sabo RT, Hall N, Foreman A, Schubert PL, Gallagher GR, Fink T, Madoff LC, Gabriel SB, MacInnis B, Park DJ, Siddle KJ, Harik V, Arvidson D, Brock-Fisher T, Dunn M, Kearns A, Laney AS: Outbreak of SARS-CoV-2 infections, including COVID-19 vaccine breakthrough infections, associated with large public gatherings-Barnstable County, Massachusetts, July 2021. MMWR Morb Mortal Wkly Rep 2021, 70:1059-1062

32. Chia PY, Xiang Ong SW, Chiew CJ, Ang LW, Chavatte J-M, Mak TM, Cui L, Kalimuddin S, Chia WN, Tan CW, Ann Chai LY, Tan SY, Zheng S, Pin Lin RT, Wang L, Leo Y-S, Lee VJ, Lye DC, Young BE: Virological and serological kinetics of SARS-CoV-2 Delta variant vaccine-breakthrough infections: a multi-center cohort study. medRxiv 2021, [Preprint] doi: 10.1101/2021.07.28.21261295

33. Sheikh A, McMenamin J, Taylor B, Robertson C; Public Health Scotland and the EAVE II Collaborators: SARS-CoV-2 Delta VOC in Scotland: demographics, risk of hospital admission, and vaccine effectiveness. Lancet 2021, 397:2461-2462

34. Riemersma KK, Grogan BE, Kita-Yarbro A, Halfmann PJ, Segaloff HE, Kocharian A, Florek KR, Westergaard R, Bateman A, Jeppson GE, Kawaoka Y, O'Connor DH, Friedrich TC, Grande KM: Shedding of infectious SARS-CoV-2 despite vaccination. medRxiv 2021, [Preprint] doi: 10.1101/2021.07.31.21261387

35. Bergwerk M, Gonen T, Lustig Y, Amit S, Lipsitch M, Cohen C, Mandelboim M, Gal Levin E, Rubin C, Indenbaum V, Tal I, Zavitan M, Zuckerman N, Bar-Chaim A, Kreiss Y, Regev-Yochay G: Covid-19 breakthrough infections in vaccinated health care workers. N Engl J Med 2021, 385:1474-1484

36. Goldberg $\mathrm{Y}$, Mandel $\mathrm{M}$, Bar-On YM, Bodenheimer $\mathrm{O}$, Freedman L, Haas EJ, Milo R, Alroy-Preis S, Ash N, Huppert A: Waning immunity of the BNT162b2 vaccine: a nationwide study from Israel. medRxiv 2021, [Preprint] doi: 10.1101/2021.08.24. 21262423 